\title{
Calculation of the Nonlinear Susceptibility in van der Waals Crystals
}

\author{
Mingxi Chen, Chao Tang, Tadao Tanabe*, Yutaka Oyama \\ Department of Materials Science, Graduate School of Engineering, Tohoku University, Sendai, Japan \\ Email: *tadao.tanabe.b1@tohoku.ac.jp
}

How to cite this paper: Chen, M.X., Tang, C., Tanabe, T. and Oyama, Y. (2019) Calculation of the Nonlinear Susceptibility in van der Waals Crystals. Optics and Photonics Journal, 9, 178-188.

https://doi.org/10.4236/opj.2019.911016

Received: August 28, 2019

Accepted: November 15, 2019

Published: November 18, 2019

Copyright $\odot 2019$ by author(s) and Scientific Research Publishing Inc. This work is licensed under the Creative Commons Attribution International License (CC BY 4.0).

http://creativecommons.org/licenses/by/4.0/

\begin{abstract}
The development of theoretical models for crystals has led to the evolution of computational methods with which much more thorough investigations than previously possible can be done, including studies of the nonlinear optical properties. There has recently been a rise in interest in 2-dimensional materials; unfortunately, measurements of the nonlinear susceptibility of these materials in the wavelength range of the order of hundreds of nanometers by traditional methods are difficult. Studies of second-harmonic generation (SHG) from the transition-metal dichalcogenides (TMDCs), $\mathrm{MoS}_{2}$ and $\mathrm{MoSe}_{2}$, have been reported; however, SHG from other typical van der Waals crystals such as GaSe and other transition metal monochalcogenides (TMMCs) has rarely been studied under the same conditions. In this study, the $211(\mathrm{i}=2, \mathrm{j}$ $=1, \mathrm{k}=1$ ) elements in the susceptibility matrices of GaSe, InSe, $\mathrm{MoS}_{2}$ and $\mathrm{WS}_{2}$ were calculated and compared. A tendency for the SHG intensity to weaken as the wavelength increases from $500 \mathrm{~nm}$ to $1000 \mathrm{~nm}$ was observed for GaSe and InSe, and, apart from some periodic fluctuations, no clear increase could be seen for these two materials in the SHG response curve in the near infrared. By comparison, $\mathrm{MoS}_{2}$ and $\mathrm{WS}_{2}$ have obvious peaks in both the visible and infrared bands. Calculations of the SHG response show peaks at around $500 \mathrm{~nm}$ (for GaSe), 570 (for InSe), $660 \mathrm{~nm}, 980 \mathrm{~nm}$ (for $\mathrm{MoS}_{2}$ ) and $580 \mathrm{~nm}, 920 \mathrm{~nm}$ (for $\mathrm{WS}_{2}$ ). Moreover, similarities between the SHG curves for GaSe and InSe and for $\mathrm{MoS}_{2}$ and WS $\mathrm{W}_{2}$ were revealed, which may be due to the similarities found for these two groups of crystals.
\end{abstract}

\section{Keywords}

Two-Dimensional Material, Second-Harmonic Generation, Density Functional Theory, Density Functional Perturbation Theory

\section{Introduction}

Because of their high mobility and thermal conductivity, and their good me- 
chanical properties, the interest in van der Waals crystals has increased in recent decades. In addition to graphene, chalcogenide compound semiconductors with two-dimensional sublayer structures have been prepared and studied [1] [2] [3] [4] [5], and the band characteristics of these compound materials make it possible for them to be applied for light-emission and detection, communication, imaging and other related functions [6] [7] [8]. Moreover, due to the special symmetry of van der Waals crystals, which arises from the sublayers, they are considered to be ideal nonlinear-optical materials, and this has attracted interest among researchers. The nonlinear optical response of materials with different thicknesses has been studied using several methods. Taking a monolayer of $\mathrm{MoS}_{2}$ as an example, a strong resonant SHG coefficient was obtained which was attributed to electronic structural changes at the layer edges [9] [10], and it has been reported that as the number of layers increases, the position and intensity of the SHG response curve shifts. Some attempts at applying van der Waals crystals as frequency modulation devices have been made and the prospect of using them, especially in communication, has been predicted [8] [11] [12] [13] [14] [15].

Other nonlinear optical effects are the intensity of the SHG response with respect to the polarization of the light incident on the crystal in a particular electric field; thus, the SHG response is considered to be a function of the incident light frequency. It is important to find the optimal nonlinear optical crystal for a particular frequency band. Although efforts have been made on investigating nonlinear optical properties, these have been limited by the high expenditure required for crystal growth and the construction of the optical system; thus, experimental investigations of a huge number of crystals in order to compare the nonlinear coefficients of each material is costly and inefficient. In the last decade, due to the development of theoretical models and computational methods, some results, such as the susceptibilities of TMDCs, have been obtained from first principles calculations. More specifically, calculations for $\mathrm{MoS}_{\mathrm{x}} \mathrm{Se}_{2-\mathrm{x}}$ were reported by Hu et al. and an interpretation of the SHG disparity at $810 \mathrm{~nm}$ was put forward [16] [17]. Though efforts have been made, the SHG properties at wavelengths above a hundred nanometers of other typical van der Waals crystals including the transition metal monochalcogenides (TMMDs), GaSe, InSe, have rarely been studied and still need to be investigated [18] [19] and in this study we calculated and directly compared the susceptibilities of 4 kinds of typical transition metal chalcogenides ( GaSe, InSe, $\left.\mathrm{MoS}_{2}, \mathrm{WS}_{2}\right)$. Considering the practical applications, we chose the bulk form of typical van der Waals crystals to do the calculation, which is also different from previous research [16].

This study theoretically predict the SHG response of crystals in the 500 to $1100 \mathrm{~nm}$ wavelength range by using the calculations, which are mainly based on density functional theory (DFT) and density functional perturbation theory (DFPT) with ab initio application of the $2 n+1$ theorem [20] [21] [22]. GaSe, 
InSe, $\mathrm{MoS}_{2}$ and $\mathrm{WS}_{2}$ were chosen as representatives not only because they are typical van der Waals crystals but also because their structural similarities allow them to be divided into two groups- $\mathrm{MX}$ and $\mathrm{MX}_{2}$, where $\mathrm{M}$ represents the metal ( $\mathrm{Ga}, \mathrm{In}, \mathrm{Mo}$ ) and $\mathrm{X}$ is the chalcogen ( $\mathrm{S}, \mathrm{Se})$. With our calculations, it is possible to filter out inappropriate candidates, and find the best optical crystal needed for a given frequency band.

\section{Theoretical}

According to the Hohenberg and Kohn theorem, all the physical properties of an electronic system can be uniquely determined by its ground-state charge density distribution [23]. Thus, getting information about the ground-state is the very first step in calculating the susceptibility. Density functional theory states that the system energy can be written as a functional of charge density and that the ground-state energy functional has the minimum value [24] [25]. The explicit form of the energy functional can be expressed in terms of Kohn Sham eigenvalues as below [23].

$$
E[n]=2 \sum_{N=1}^{N / 2} \epsilon_{n}-\frac{e^{2}}{2} \int \frac{n(\boldsymbol{r}) n\left(\boldsymbol{r}^{\prime}\right)}{\left|\boldsymbol{r}-\boldsymbol{r}^{\prime}\right|} \mathrm{d} \boldsymbol{r} \mathrm{d} \boldsymbol{r}^{\prime}+E_{x c}[n]-\int n(\boldsymbol{r}) v_{x c}(\boldsymbol{r}) \mathrm{d} \boldsymbol{r}
$$

Here $N$ is the number of electrons, $-e$ is the electron charge, $n(r)$ is the charge density at position $\boldsymbol{r}, E_{x c}$ is the exchange-correlation energy, $v_{x c}$ is defined as $v \equiv \delta E / \delta n(\boldsymbol{r})$ and $\epsilon_{n}$ is the Kohn Sham eigenvalue corresponding to the nth state. In the formula we have divided the system energy into three parts, and the exchange-correlation term, $E_{x c}$ is known to be the smallest, but cannot be ignored. Since an exact expression for the exchange-correlation energy is unknowable, Kohn and Sham proposed a reasonable approximation for it. It is considered that the exchange-correlation energy for a real electron is equal to the $E_{x c}$ in a homogeneous electron gas with the same charge-density distribution, and the assumption is known as the local density approximation (LDA) [26]. Although the LDA is a really successful approach in most cases, it also well known that this method underestimates the bandgaps of materials [23] [27]. For this reason, scissors-type bandgap corrections are needed in our calculations and the specific values used will be discussed later.

The second-harmonic generation corresponds to the third-order derivative of the system energy, but due to the $2 n+1$ theorem, the first-order derivative of the wave function is sufficient to calculate the SHG response, and the susceptibility is as follows:

$$
\chi^{(2)}=3 i \frac{e \Omega}{2 \pi \boldsymbol{E}_{0}^{2}} \sum_{m, n=1}^{N} \int_{B Z} \mathrm{~d} k \times\left\langle u_{m}^{k}\left|\frac{\partial}{\partial k}\left(\left|u_{n}^{k}\right\rangle\left\langle P_{c} u_{n}^{k(1)}\right|\right)\right| P_{c} u_{m}^{k(1)}\right\rangle
$$

where $\Omega$ is the dimensions of the unit cell and $u$ is the periodic part of the Bloch wave function, $E_{0}$ is the external electric field, and $P_{c}$ is the state projection operator [23]. The first derivative of the wavefunction can be obtained from perturbation theory. 


\section{Calculational Details}

Details of the structural parameters of GaSe, InSe, $\mathrm{MoS}_{2}$ and $\mathrm{WS}_{2}$ used in the calculations are shown in Table 1 and Figure 1 [28] [29] [30] [31] [32].

Norm-conserving Fritz-Haber pseudopotentials with a cutoff energy of 100Ry were used in our calculations, which was deemed to cover the states needed to calculate the SHG response. The sampling point densities of the $\mathrm{GaSe}$, InSe, $\mathrm{MoS}_{2}$ and $\mathrm{WS}_{2}$ Brillouin zones were set to $10 \times 10 \times 10,7 \times 7 \times 7,8 \times 8 \times 8,8 \times 8$ $\times 8$ respectively.

Table 1. Primitive vectors and coordinates of the basis atoms of GaSe, InSe, $\mathrm{MoS}_{2}$ and $\mathrm{WS}_{2}$, in which $t_{1}, t_{2}, t_{3}$ represent the base vectors in Cartesian coordinate system used to decribe the unit cells. $a$ and $c$ are lattice consistants. $\mathrm{X}_{\mathrm{y}}$ represents the coordinate in $\left(t_{1}, t_{2}, t_{3}\right)$ of $\mathrm{X}$-atom in $\mathrm{y}$-sublayer.

\begin{tabular}{|c|c|}
\hline GaSe & InSe \\
\hline$t_{1}=(-a, 0,0)$ & $t_{1}=(-a, 0,0)$ \\
\hline$t_{2}=(-a / 2, a \sqrt{3} / 2,0)$ & $t_{2}=(-a / 2, a \sqrt{3} / 2,0)$ \\
\hline$t_{3}=(0,0, c)$ & $t_{3}=(0,0, c)$ \\
\hline$a=3.755 \AA ̊, c=15.94 \AA$ & $a=4.05 \AA ̊, c=16.64 \AA$ \\
\hline $\mathrm{Se}_{1}:(1 / 3,2 / 3,0.1003)$ & $\mathrm{Se}_{1}:(1 / 3,2 / 3,0.0907)$ \\
\hline $\mathrm{Ga}_{1}:(0,0,0.1701)$ & $\mathrm{In}_{1}:(0,0,0.1677)$ \\
\hline $\mathrm{Ga}_{\text {II }}:(0,0,0.3299)$ & $\mathrm{In}_{\mathrm{II}}:(0,0,0.3233)$ \\
\hline $\mathrm{Se}_{\mathrm{II}}:(1 / 3,2 / 3,0.3997)$ & $\mathrm{Se}_{\mathrm{II}}:(1 / 3,2 / 3,0.4093)$ \\
\hline $\mathrm{Se}_{\mathrm{III}}:(2 / 3,1 / 3,0.6003)$ & $\mathrm{Se}_{\mathrm{III}}:(2 / 3,1 / 3,0.5907)$ \\
\hline $\mathrm{Ga}_{\mathrm{III}}:(1 / 3,2 / 3,0.6701)$ & $\mathrm{In}_{\mathrm{III}}:(1 / 3,2 / 3,0.6677)$ \\
\hline $\mathrm{Ga}_{\mathrm{IV}}:(1 / 3,2 / 3,0.8299)$ & $\mathrm{In}_{\mathrm{IV}}:(1 / 3,2 / 3,0.8323)$ \\
\hline $\mathrm{Se}_{\mathrm{IV}}:(2 / 3,1 / 3,0.8997)$ & $\mathrm{Se}_{\mathrm{IV}}:(2 / 3,1 / 3,0.9093)$ \\
\hline $\mathrm{MoS}_{2}$ & $\mathrm{WS}_{2}$ \\
\hline$t_{1}=(-a, 0,0)$ & $t_{1}=(-a, 0,0)$ \\
\hline$t_{2}=(-a / 2, a \sqrt{3} / 2,0)$ & $t_{2}=(-a / 2, a \sqrt{3} / 2,0)$ \\
\hline$t_{3}=(0,0, c)$ & $t_{3}=(0,0, c)$ \\
\hline$a=3.122 \AA, c=12.32 \AA$ & $a=3.153 \AA, c=12.323 \AA$ \\
\hline$S_{I}:(1 / 3,2 / 3,0.1250)$ & $S_{1}:(1 / 3,2 / 3,0.0907)$ \\
\hline $\mathrm{Mo}_{1}:(0,0,0.2500)$ & $\mathrm{W}_{\mathrm{I}}:(0,0,0.1677)$ \\
\hline $\mathrm{S}_{\mathrm{II}}:(1 / 3,2 / 3,0.3750)$ & $\mathrm{S}_{\mathrm{II}}:(1 / 3,2 / 3,0.4093)$ \\
\hline $\mathrm{S}_{\mathrm{III}}:(2 / 3,1 / 3,0.6250)$ & $\mathrm{S}_{\mathrm{III}}:(2 / 3,1 / 3,0.5097)$ \\
\hline $\mathrm{Mo}_{\text {III }}:(1 / 3,2 / 3,0.7500)$ & $\mathrm{W}_{\mathrm{III}}:(1 / 3,2 / 3,0.6677)$ \\
\hline $\mathrm{S}_{\mathrm{IV}}:(2 / 3,1 / 3,0.8750)$ & $\mathrm{S}_{\mathrm{IV}}:(2 / 3,1 / 3,0.9093)$ \\
\hline
\end{tabular}




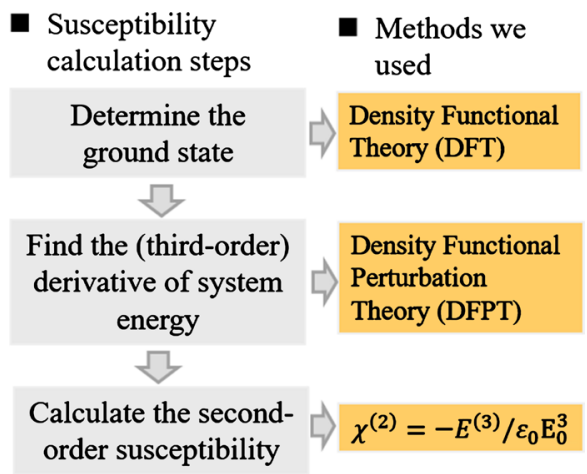

Figure 1. Calculation steps with the methods we applied in each steps.

\section{Results}

\subsection{Band Structure Verification}

To verify the accuracy of the calculated electronic structures, we compared the band diagrams obtained with ones published in previous papers. Underestimates of the bandgaps were revealed by the comparison. Here, the band structures of GaSe InSe $\mathrm{MoS}_{2}$ and $\mathrm{WS}_{2}$ are given in Figure 2(a)-(d) respectively. By comparison with results published previously by other researchers, the accuracy of the calculations was determined and the scissors corrections obtained. These are given in Table 2 and Figure 3.

\subsection{Nonlinear Optical Tensor}

As we know, the second-order susceptibility matrix has $3^{3}=27$ elements. For GaSe, InSe, $\mathrm{MoS}_{2}$, and $\mathrm{WS}_{2}$, the 211 element is the one that concerns us most: most of the other elements are canceled to zero due to the symmetry of these crystals, and coaxial phase matching is non-negligible in nonlinear optical processes. The susceptibility is a complex number, in which the real part represents the light arising, and information about the energy loss is obtained from the imaginary part combined with the real part. The absolute values of the 211 element of the susceptibility matrix, which is proportional to the SHG emergent light intensity is given in Figure 4.

In Figure 4, it is can be seen that all four materials have significant SHG effects in the visible and infrared bands, and at particular wavelengths the nonlinear optical response is several orders of magnitude larger than the band. These results are consistent with previous results. Moreover, crystals with similar structures have similar nonlinear response curves, with a short-wave-directed shift as the actual bandgap increases. The phenomenon is understandable and the result is implied by the scissors corrections obtained.

In particular, the nonlinear responses of all four crystals in the green to red light region of the visible light band reach significant levels, with InSe, GaSe and $\mathrm{MoS}_{2}$ having their highest response efficiency in this region. The results predict that the nonlinear response will give rise to peaks at $500 \mathrm{~nm}, 570 \mathrm{~nm}, 660 \mathrm{~nm}$ and $570 \mathrm{~nm}$ for the four kinds of material. As the wavelength increases, the 
(a)

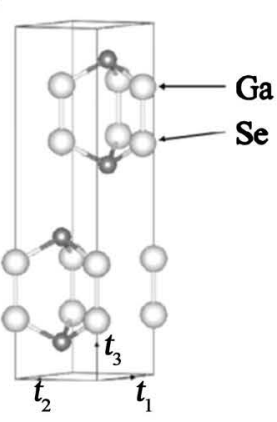

(b)

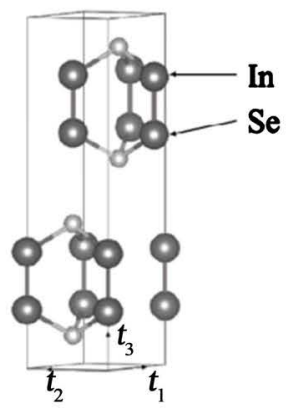

(c)

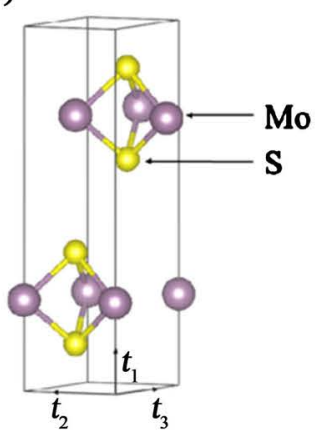

(d)

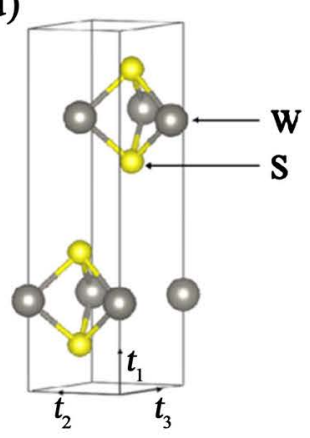

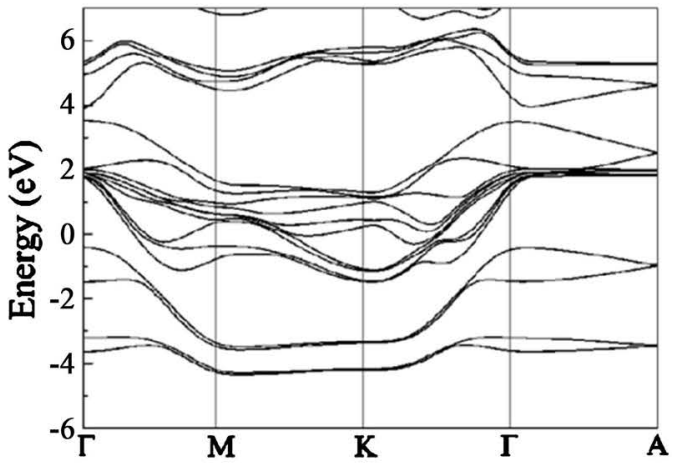
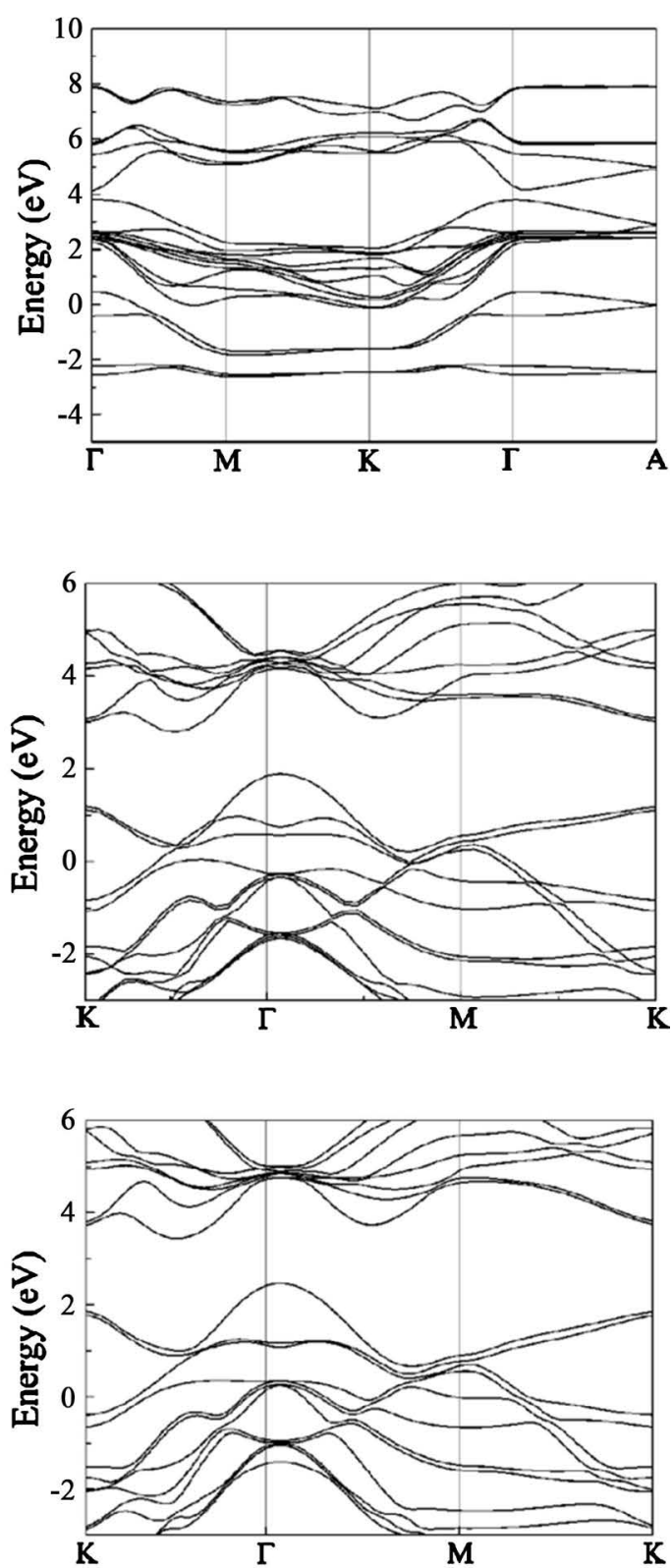

Figure 2. Unit cell and band structures of van der Waals crystals for (a) GaSe, (b) InSe (c) $\mathrm{MoS}_{2}$ and (d) $\mathrm{WS}_{2}$, respectively. 
Table 2. Bandgaps obtained by our calculation, actual size of the gaps and the scissors corrections obtained by comparing the values.

\begin{tabular}{cccc}
\hline Material & Bandgap obtained by calculation & Actual size of Bandgap & Scissor correction \\
\hline GaSe & $0.57 \mathrm{eV}$ & $2.01 \mathrm{eV} \mathrm{[29]}$ & $1.44 \mathrm{eV}$ \\
InSe & $0.33 \mathrm{eV}$ & $1.31 \mathrm{eV} \mathrm{[33]}$ & $0.98 \mathrm{eV}$ \\
$\mathrm{MoS}_{2}$ & $0.91 \mathrm{eV}$ & $1.23 \mathrm{eV} \mathrm{[34]}$ & $0.31 \mathrm{eV}$ \\
$\mathrm{WS}_{2}$ & $0.97 \mathrm{eV}$ & $1.32 \mathrm{eV} \mathrm{[35]}$ & $0.35 \mathrm{eV}$ \\
\hline
\end{tabular}
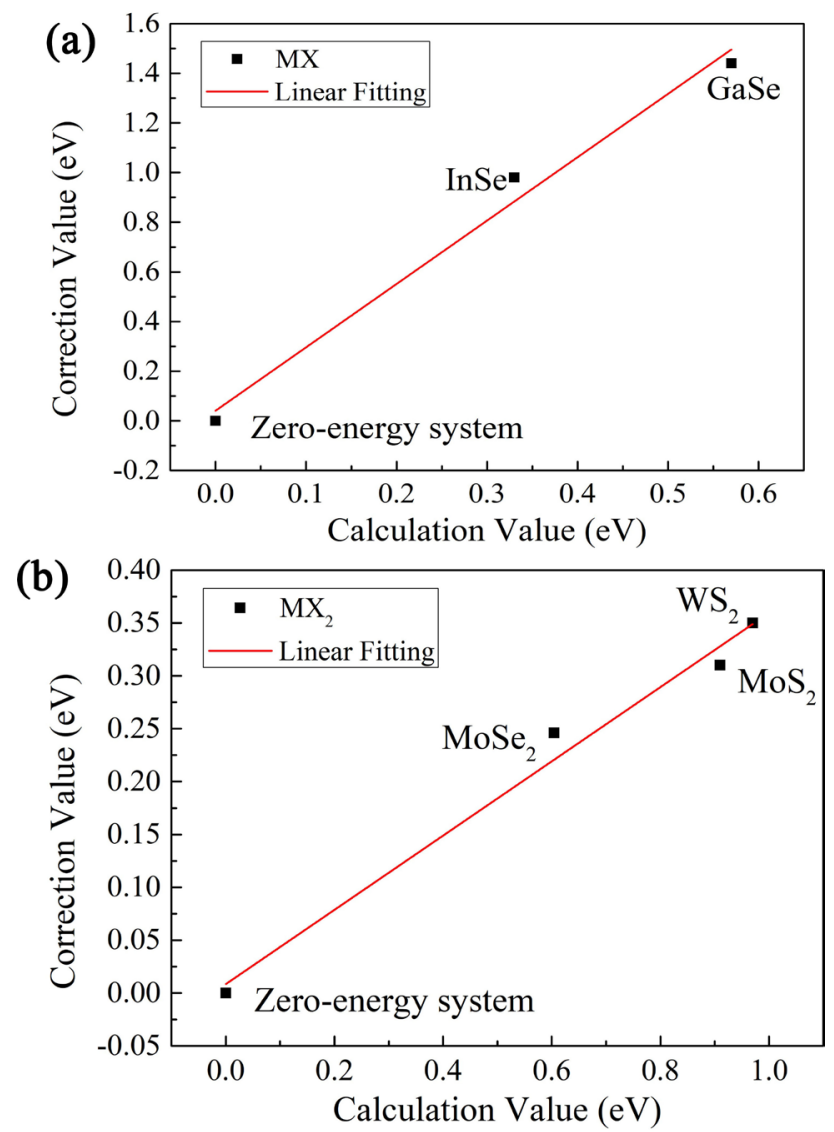

Figure 3. Scissors correction values for the bandgaps obtained by comparing actual bandgaps with the values obtained by calculation using DFT with LDA for (a) AB-structured materials like GaSe and (b) $\mathrm{AB}_{2}$-structured materials like $\mathrm{MoS}_{2}$, respectively. Besides the parameters we need for later discussion, the points for $\mathrm{MoSe}_{2}$ and the zero energy system are shown in the plots.

intensity of the SHG response decreases and is weaker for GaSe and InSe, but we can still see some small periodic peaks in the spectral distribution as shown in Figure 3. In contrast, $\mathrm{WS}_{2}$ and $\mathrm{MoS}_{2}$ are deemed to be better choices if an IR-band SHG device is needed. Specifically, we can expect to see clear SHG peaks at around $980 \mathrm{~nm}$ for $\mathrm{MoS}_{2}$ and $920 \mathrm{~nm}$ for $\mathrm{WS}_{2}$. However, in the DFT and DFPT calculations, the effects of thermal vibrations in the lattice and defects are not included, and these may give rise to discrepancies between our calculations and the results of experiments. 


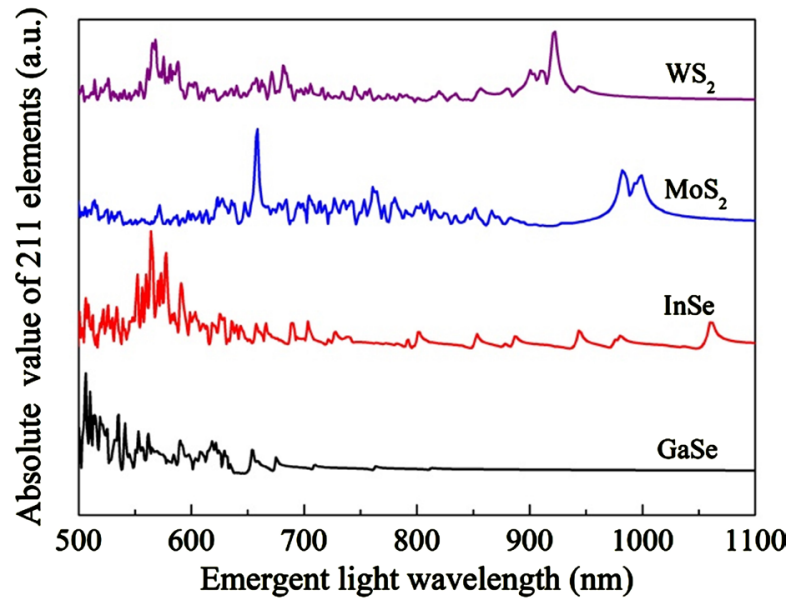

Figure 4. The absolute value of the spectral distribution of the 211 element in the (2nd order) susceptibility matrices calculated by DFT method of (a) GaSe, (b) InSe, (c) $\mathrm{MoS}_{2}$ and (d) $\mathrm{WS}_{2}$ in the wavelength range from $500 \mathrm{~nm}$ to $1100 \mathrm{~nm}$, respectively.

\section{Conclusion}

We used first principles calculations to obtain the SHG transition efficiencies of four typical van der Waals crystals, including the TMMCs, $\mathrm{MoS}_{2}$ and $\mathrm{WS}_{2}$, and the TMDCs, GaSe and InSe. From our calculations we obtained a linear regression for the scissors correction, and similar SHG response curves were observed for the two groups of materials, which may be due to the similarities between the crystal structures and their electronic states. Our calculations can be used to predict the SHG response of several crystals in the 500 to $1100 \mathrm{~nm}$ wavelength range, and so enable unsuitable nonlinear optical materials to be filtered out. SHG from GaSe and InSe in the visible light region is predicted. The calculations predict that high peaks for light emerging at $500 \mathrm{~nm}$ (for GaSe) and $570 \mathrm{~nm}$ (for InSe) would be observed. Moreover, two high SHG response regions in the visible light and infrared regions for $\mathrm{MoS}_{2}$ and $\mathrm{WS}_{2}$ are predicted by the calculations. Peaks in the SHG transformation efficiency at $660 \mathrm{~nm}$ and $980 \mathrm{~nm}$ for $\mathrm{MoS}_{2}$ and at $580 \mathrm{~nm}$ and $920 \mathrm{~nm}$ for $\mathrm{WS}_{2}$ were obtained. In the future, the present calculation will be extended to other $2 \mathrm{D}$ materials such as graphene, to find their potentials of nonlinear optical application.

\section{Acknowledgements}

This work was partly supported by Grant-in-Aid for JSPS Research Fellow JP19J20564, Japan.

\section{Conflicts of Interest}

The authors declare no conflicts of interest regarding the publication of this paper.

\section{References}

[1] Gouskov, A., Camassel, J. and Gouskov, L. (1982) Growth and Characterization of 
III-VI Layered Crystals like GaSe, GaTe, InSe, GaSe $e_{1-\mathrm{x}} \mathrm{Te}_{\mathrm{x}}$ and GaxIn ${ }_{1-\mathrm{x}}$ Se. Progress in Crystal Growth and Characterization of Materials, 5, 323. https://doi.org/10.1016/0146-3535(82)90004-1

[2] Shaw, J. C., Zhou, H., Chen, Y., Weiss, N. O., Liu, Y., Huang, Y. and Duan, X. (2014) Chemical Vapor Deposition Growth of Monolayer MoSe ${ }_{2}$ Nanosheets. Nano Research, 7, 511. https://doi.org/10.1007/s12274-014-0417-z

[3] Tanabe, T., Zhao, S., Sato, Y. and Oyama, Y. (2017) Effect of Adding Te to Layered GaSe Crystals to Increase the van der Waals Bonding Force. Journal of Applied Physics, 122, Article ID: 165105. https://doi.org/10.1063/1.4986768

[4] Oyama, Y., Tanabe, T., Sato, F., Kenmochi, A., Nishizawa, J., Sasaki, T. and Suto, K. (2008) Liquid-Phase Epitaxy of GaSe and Potential Application for Wide Frequency-Tunable Coherent Terahertz-Wave Generation. Journal of Crystal Growth, 310, 1923. https://doi.org/10.1016/j.jcrysgro.2007.11.205

[5] Tanabe, T., Tang, C., Sato, Y. and Oyama, Y. (2018) Direct Determination of the Interlayer van der Waals Bonding Force in 2D Indium Selenide Semiconductor Crystal. Journal of Applied Physics, 123, Article ID: 245107. https://doi.org/10.1063/1.5024313

[6] Takasuna, S., Shiogai, J., Matsuzaka, S., Kohda, M., Oyama, Y. and Nitta, J. (2017) Effect of Optical Waveguide on Photoluminescence Polarization in Layered Material GaSe with Millimeter Scale. Physical Review B, 96, Article ID: 161303. https://doi.org/10.7567/JJAP.57.020308

[7] Auerbach, S.M., Carrado, K.A. and Dutta, P.K. (2004) Handbook of Layered Materials. CRC Press, New York. https://doi.org/10.1201/9780203021354

[8] Kenmochi, A., Tanabe, T., Oyama, Y., Suto, K. and Nishizawa, J. (2008) Terahertz Wave Generation from GaSe Crystals and Effects of Crystallinity. Journal of Physics and Chemistry of Solids, 69, 605. https://doi.org/10.1016/j.jpcs.2007.07.047

[9] Neshev, D. and Kivshar, Y. (2014) Nonlinear Optics Pushed to the Edge. Science, 344, 483. https://doi.org/10.1126/science.1253531

[10] Yin, X., Ye, Z., Chenet, D.A., Ye, Y., O’Brien, K., Hone, J.C. and Zhang, X. (2014) Edge Nonlinear Optics on a $\mathrm{MoS}_{2}$ Atomic Monolayer. Science, 344, 488.

https://doi.org/10.1126/science.1250564

[11] Geim, A.K. and Grigorieva, I.V. (2013) Van der Waals Heterostructures. Nature, 499, 419. https://doi.org/10.1038/nature12385

[12] Coleman, J.N., Lotya, M., O’Neill, A., Bergin, S.D., King, P.J., et al. (2011) TwoDimensional Nanosheets Produced by Liquid Exfoliation of Layered Materials. Science, 331, 568. https://doi.org/10.1126/science.1194975

[13] Tang, C., Sato, Y., Tanabe, T. and Oyama, Y. (2018) Low Temperature Liquid Phase Growth of Crystalline InSe Grown by the Temperature Difference Method under Controlled Vapor Pressure. Journal of Crystal Growth, 495, 54. https://doi.org/10.1016/j.jcrysgro.2018.05.016

[14] Eaton, D.F., Meredith, G.R. and Miller, J.S. (1992) Molecular Nonlinear Optical Materials-Potential Applications. Advanced Materials, 4, 45. https://doi.org/10.1002/adma.19920040110

[15] Li, D.Q., Ratner, M.A. and Marks, T.J. (1988) Molecular and Macromolecular Nonlinear Optical Materials. Probing Architecture/Electronic Structure/Frequency Doubling Relationships via an SCF-LCAO MECI. pi. Electron Formalism. Journal of the American Chemical Society, 110, 1707. https://doi.org/10.1021/ja00214a008

[16] Hu, L., Wei, D. and Huang, X. (2017) Second Harmonic Generation Property of 
Monolayer TMDCs and Its Potential Application in Producing Terahertz Radiation. The Journal of Chemical Physics, 147, Article ID: 244701. https://doi.org/10.1063/1.5000561

[17] Wang, C.-Y. and Guo, G.-Y. (2015) Nonlinear Optical Properties of Transition-Metal Dichalcogenide MX2 ( $\mathrm{M}=\mathrm{Mo}, \mathrm{W} ; \mathrm{X}=\mathrm{S}, \mathrm{Se})$ Monolayers and Trilayersfrom First-Principles Calculations. The Journal of Physical Chemistry C, 119, 13268-13276. https://doi.org/10.1021/acs.jpcc.5b01866

[18] Cabuk, S. (2012) The Nonlinear Optical Susceptibility and Electro-Optic Tensor of Ferroelectrics: First-Principle Study. Central European Journal of Physics, 10, 239. https://doi.org/10.2478/s11534-011-0079-3

[19] Sharma, S. and Ambrosch-Draxl, C. (2004) Second-Harmonic Optical Response from First Principles. Physica Scripta, 109, 128. https://doi.org/10.1238/Physica.Topical.109a00128

[20] Gonze, X., Amadon, B., Anglade, P.-M., Beuken, J.-M., Bottin, F., et al. (2009) ABINIT: First-Principles Approach to Material and Nanosystem Properties. Computer Physics Communications, 180, 2582. https://doi.org/10.1016/j.cpc.2009.07.007

[21] Gonze, X., Rignanese, G.-M., Verstraete, M., Beuken, J.-M., Pouillon, Y., Caracas, R., Jollet, F., Torrent, M., Zerah, G., Mikami, M., Ghosez, Ph., Veithen, M., Raty, J.-Y., Olevano, V., Bruneval, F., Reining, L., Godby, R., Onida, G., Hamann, D.R. and Allan, D.C. (2005) A Brief Introduction to the ABINIT Software Package. Zeitschrift für Kristallographie, 220, 558.

[22] Gonze, X. (2002) First-Principles Computation of Material Properties: The ABINIT Software Project. Computational Materials Science, 25, 478.

https://doi.org/10.1016/S0927-0256(02)00325-7

[23] Baroni, S., De Gironcoli, S., Dal Corso, A. and Giannozzi, P. (2001) Phonons and Related Crystal Properties from Density-Functional Perturbation Theory. Reviews of Modern Physics, 73, 515. https://doi.org/10.1103/RevModPhys.73.515

[24] Gross, E.K. and Dreizler, R.M. (1990) Density Functional Theory. Springer, Berlin. https://doi.org/10.1016/S0065-3276(08)60600-0

[25] Parr, R.G. and Yang, W. (1989) Density-Functional Theory of Atoms and Molecules. Oxford University Press, Oxford.

[26] Kohn, W. (1965) Self-Consistent Equations Including Exchange and Correlation Effects. Physical Review, 140, A1133. https://doi.org/10.1103/PhysRev.140.A1133

[27] Assadi, M.H.N. and Hanaor, D.A. (2013) Theoretical Study on Copper's Energetics and Magnetism in $\mathrm{TiO}_{2}$ Polymorphs. Journal of Applied Physics, 113, Article ID: 233913. https://doi.org/10.1063/1.4811539

[28] Nagel, S., Baldereschi, A. and Maschke, K. (1979) Tight-Binding Study of the Electronic States in GaSe Polytypes. Journal of Physics C: Solid State Physics, 12, 1625. https://doi.org/10.1088/0022-3719/12/9/006

[29] Plucinski, L., Johnson, R., Kowalski, B., Kopalko, K., Orlowski, B., Kovalyuk, Z. and Lashkarev, G. (2003) Electronic Band Structure of GaSe (0001): Angle-Resolved Photoemission and ab Initio Theory. Physical Review B, 68, Article ID: 125304. https://doi.org/10.1103/PhysRevB.68.125304

[30] McCanny, J. and Murray, R. (1977) The Band Structures of Gallium and Indium Selenide. Journal of Physics C: Solid State Physics, 10, 1211. https://doi.org/10.1088/0022-3719/10/8/022

[31] Kadantsev, E.S. and Hawrylak, P. (2012) Electronic Structure of a Single $\mathrm{MoS}_{2}$ Mo- 
nolayer. Solid State Communications, 152, 909.

https://doi.org/10.1016/j.ssc.2012.02.005

[32] Schutte, W., De Boer, J. and Jellinek, F. (1987) Crystal Structures of Tungsten Disulfide and Diselenide. Journal of Solid State Chemistry, 70, 207.

https://doi.org/10.1016/0022-4596(87)90057-0

[33] Debbichi, L., Eriksson, O. and Lebègue, S. (2015) Two-Dimensional Indium Selenides Compounds: An ab Initio Study. The Journal of Physical Chemistry Letters, 6 , 3098. https://doi.org/10.1021/acs.jpclett.5b01356

[34] Kobayashi, K. and Yamauchi, J. (1995) Electronic Structure and Scanning-Tunneling-Microscopy Image of Molybdenum Dichalcogenide Surfaces. Physical Review B, 51, Article ID: 17085. https://doi.org/10.1103/PhysRevB.51.17085

[35] Albe, K. and Klein, A. (2002) Density-Functional-Theory Calculations of Electronic Band Structure of Single-Crystal and Single-Layer WS 2. Physical Review B, 66, Article ID: 073413. https://doi.org/10.1103/PhysRevB.66.073413 